\title{
Jährliches Thorax-Röntgen reduziert Lungenkrebs-Mortalität nicht
}

\section{Die Erfolgsgeschichte der Früher- kennung des Kolonkarzinoms lässt sich leider nicht auf das Bronchial- karzinom übertragen. Auch jährliche Röntgenuntersuchungen beeinflus- sen die kumulative Mortalität des Bronchialkarzinoms nicht.}

- Das Bronchialkarzinom ist nach wie vor die führende Krebs-Todesursache weltweit. In den siebziger und achtziger Jahren konnten Studien keinen positiven Effekt eines Screenings mit der Sputum-Zytologie oder dem RöntgenThorax zeigen. In einer Studie aus jüngster Zeit gelang es allerdings, mittels regelmäßigem Screening mit der Computertomografie des Thorax die Mortalität am Bronchialkarzinom um 20\% zu senken.

Zwischen November 1993 und Juli 2001 wurden 154901 Personen im Alter zwischen 55 und 74 Jahren in eine Gruppe mit jährlichem Röntgen-Thorax-Screening und üblicher Versorgung randomisiert. Ein Röntgen-Thorax-Befund wurde im Screening als positiv gewertet, wenn ein Rundherd, eine Raumforderung, ein Infiltrat oder ein anderer Befund, der verdächtig auf ein Bronchialkarzinom war, festgestellt wurde. Das weitere diagnostische Vorgehen lag im Ermessen des behandelnden Arztes und des betroffenen Patienten. Teilnehmer der Kontrollgruppe erhielten keine Intervention und standen unter üblicher medizinischer Versorgung. Alle diagnostizierten Krebserkrankungen, Todesfälle und Todesursachen wurden bis zum 31. Dezember 2009 dokumentiert und nachverfolgt.

86,6\% der aufgeforderten Probanden erschienen zur Erstuntersuchung. Die weiteren Screening-Untersuchungen in den folgenden drei Jahren nahmen zwischen 79 und $84 \%$ der Teilnehmer wahr. In der Kontrollgruppe erhielten 11\% eine Röntgen-Thorax-Untersuchung aus verschiedenen Gründen. Die kumulative Inzidenzrate eines Bronchialkarzi-

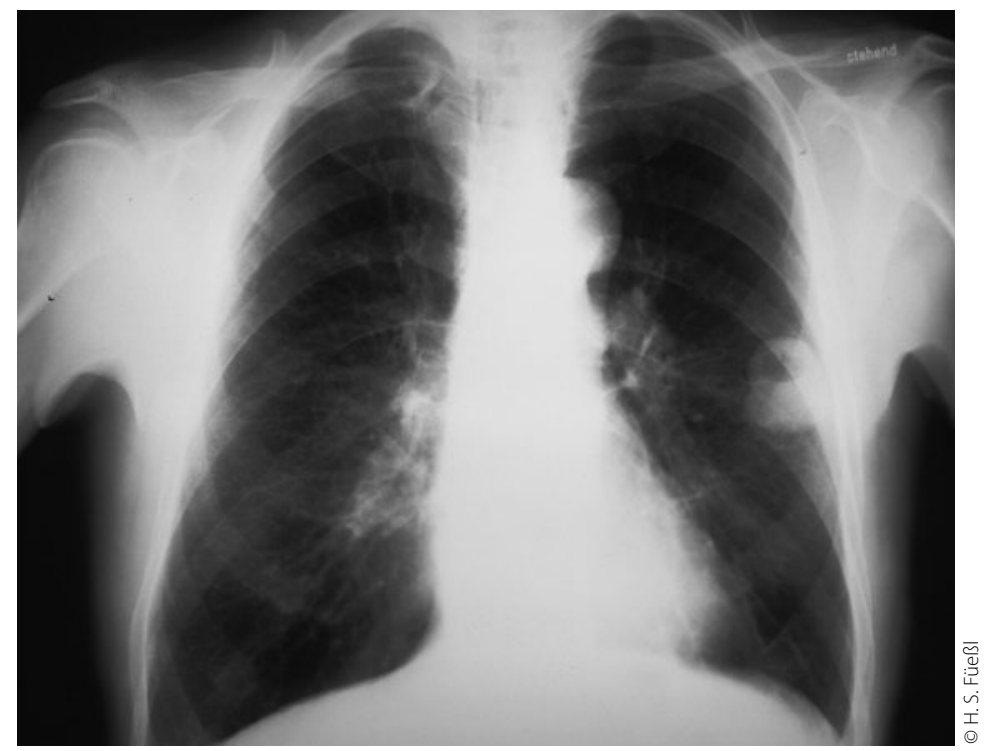

Wandnahes Bronchialkarzinom.

noms im Verlauf des 13-jährigen Follow-up betrug in der Interventionsgruppe 20,1 , in der in üblicher Weise versorgten Kohorte 19,2, jeweils pro 10000 Personenjahren. In diesem Zeitraum wurden in der Interventionsgruppe 1213, in der Kontrollgruppe 1230 Fälle eines Bronchialkarzinoms beobachtet. Das jeweilige Stadium und die Verteilung der histologischen Typen war in den beiden Gruppen weitgehend gleich. Mit dem Röntgen-Thorax-Screening gelang nicht einmal ein so genanntes „Stage Shift", d. h., es wurden nicht mehr Bronchialkarzinome in einem früheren und gut operablem Stadium erfasst als in der Kontrollgruppe.

\section{Kommentar}

Auch mit dem beträchtlichen Aufwand von vier Röntgen-Thorax-Screening-Untersuchungen im Laufe von vier Jahren gelingt es nicht, die kumulative Mortalität am Bronchialkarzinom im Lauf eines 13-jährigen Follow-up zu senken. Dieses Ergebnis ist einerseits enttäuschend, andererseits war es auch zu erwarten. Enttäuschend, weil gerade für den häufigsten malignen Tumor eine Methode der Früherkennung besonders nötig wäre; zu erwarten, weil sich vor allem das Plattenepithelkarzinom des Bronchus vorwiegend zentral manifestiert und damit auf dem Röntgenbild im Schatten des Mediastinums "verschwindet".

In diesem Zusammenhang ist auch interessant, dass die vorwiegend peripher liegenden Adenokarzinome zumindest tendenziell in den durch Screening entdeckten Fällen im Vergleich zur Kontrollgruppe überwogen. Verschattungen und Rundherde in der Lungenperipherie stellen sich eben wesentlich besser dar als zentral gelegene Befunde. Ein indirekter Beleg für diese Hypothese ist das Ergebnis einer Parallelstudie an einer Untergruppe dieser Patienten, bei der die RöntgenThorax-Untersuchung mit der Spiral-CT verglichen wurde. Mit der Computertomografie gelang es tatsächlich, das relative Risiko, am Bronchialkarzinom zu versterben, im Laufe einer sechsjährigen Beobachtungsperiode um 20\% zu senken. Dieser Aufwand ist allerdings noch bedeutend größer und erscheint auf Populationsebene kaum praktikabel zu sein - von den Kosten ganz zu schweigen.

H. S. FÜESSL =

- M. M. Oken et al.

Screening by Chest Radiograph and Lung

Cancer Mortality, JAMA 306 (2011) 1865-1873 